\title{
Extragalactic filament detection with a layer smoothing method
}

\author{
A. V. Tugay* \\ Taras Shevchenko National University of Kyiv, Glushkova ave. 4, 03127 Kyiv, Ukraine
}

\begin{abstract}
Filaments are clearly visible in galaxy distributions, but they are difficult to detect by computer algorithms. Most methods of filament detection can be used only with numerical simulations of a large-scale structure. New simple and effective methods for the real filament detection should be developed. The method of a smoothed galaxy density field was applied in this work to SDSS data of galaxy positions. Five concentric radial layers of $100 \mathrm{Mpc}$ are appropriate for filaments detection. Two methods were tested for the first layer and one more method is proposed.
\end{abstract}

Key words: cosmology: the large-scale structure of the Universe

\section{INTRODUCTION}

The cellular large-scale structure of the Universe (LSS) can be easily seen in many galaxy distributions. It is visible in the distribution of 2MASS sources on the celestial sphere and in a number of galaxy redshift surveys. Such a structure was explained in Zeldovich theory of linear growth of fluctuations, by gravitational instability. LSS is formed under the influence of gravity from the primordial dark matter fluctuations. This process leads to the formation of such elements of LSS as domain walls, filaments, galaxy clusters and voids. All these structures were simulated on computers in numerous research works. We can now describe LSS as a set of voids with galaxies between them. An average size of a void is $100 \mathrm{Mpc}$. The walls of the voids consist of one-dimensional filaments. Galaxy clusters, groups and isolated galaxies can be found in filaments. The largest clusters are located commonly on the intersections of the filaments, on the borders of two or more walls, and in voids.

In this paper we will consider the task of filament detection in a galaxy distribution. This task is necessary for the description of filaments and LSS as a whole. Further research in this realm can be useful for the dark matter studying and estimating of cosmological parameters. During the past several years a number of methods for filament detection was developed. Most of them can be used only to numerically simulate LSS, due to the fact that they need the complete information on the parameters of the distribution of all galaxies in a given volume. Only three methods were applied recently to a real galaxy distribution. This set of real galaxies can be taken from the Sloan Digital Sky Survey (SDSS). SDSS covers

\footnotetext{
* tugay . anatoliy@gmail.com

(C) A. V. Tugay, 2014
}

a large part of the sky of $120^{\circ} \times 70^{\circ}$ and has redshifts for up to one million galaxies. Although SDSS is the best galaxy sample for filament detection, application of any computer algorithm to it is quite problematic. The main problem is lack of observed galaxies in the concrete filament. This problem leads to different nonphysical artifacts in filament detection in different methods. It is not possible to recover the full network of filaments at distances above $500 \mathrm{Mpc}$, using SDSS data. This problem was solved in [2] by generating additional galaxies among the real ones, and presenting each galaxy as a complex expanded density (probability) field with its own filamentary structure. This leads to the appearance of numerous curved filaments and void bounds with an overly complex and detailed shape. Conversely, in [1], large areas in the sky were not filled by filaments. The authors found only 53 filaments in the entire SDSS volume for redshifts $z<0.15$. Up to a thousand voids and filaments should be in such a volume with the characteristic size of $100 \mathrm{Mpc}$ per void and filament. In the paper [3] filaments were detected as lines connecting nearby galaxies, groups or clusters. Real galaxy distribution has numerous spaces between galactic structures, so this method detects numerous of small filaments instead of a single large filament.

\section{METHOD}

The main idea is to look for filaments in concentric radial layers. The thickness of the layers was selected equal to the size of a void, $100 \mathrm{Mpc}$ or $7000 \mathrm{~km} / \mathrm{s}$ in radial velocity space. In a $2 \mathrm{D}$ distribution of galaxies in such layers we can easily solve the problem of filament intersection in the same position but at different distances in the sky. There should be 
no more than two filaments in the same location in a layer. Another advantage of layer consideration is the neglecting of the "finger of God" effect. Galaxies in clusters have large virial velocities, so the clusters are elongated along the line of a sight in redshift space. It is impossible to distinguish galaxies in clusters with a large velocity, from slow isolated galaxies which lie at different distances. Velocities of galaxies in clusters can reach up to $2000 \mathrm{~km} / \mathrm{s}$. But if we consider only the sky distribution of galaxies in a $7000 \mathrm{Mpc}$ thick layer, all galaxies in a cluster will fall to the same place in one layer. A cluster can be cut, of course, between two layers. We are going to solve this problem in the next works by moving the radial bounds of layers.

We will present here the results of the attempts of filament detection in the layers for a smoothed galaxy density field. The task was to fill the space between galaxies in the filament by smoothed density and leave a lower density in the voids. We used Gaussian smoothing with two parameters: dispersion and cutoff radius. In this work galaxies are considered as uniform points. In the next papers we are going to take into account such parameters of galaxies as optical and X-ray luminosity, diameter, morphological type and spatial orientation. Four methods of further analysis of a smoothed galaxy density field are considered here.

\section{DISTRIBUTION OF DENSITY MAXIMA}

The problem is to find several numerical parameters of a smoothed galaxy density field, the values of which will indicate whether the taken direction lies in a filament or not. Also, we need the parameter which will have different values in different filaments. With such parameters it will be possible to restore the network of filaments and compare it with visible distribution of galaxies in a layer. Although some filaments are visible in the layers at radial velocities up to $35000 \mathrm{~km} / \mathrm{s}$, in this paper only the first layer of SDSS galaxies is considered. Galaxies from this layer have radial velocities between 4000 and $11000 \mathrm{~km} / \mathrm{s}$. The filaments and voids of this layer are not numerous, and have the largest angular sizes, thus being the most visually detectable. This layer includes the well-known Coma cluster of galaxies. Although the filaments around the Coma cluster are the easiest to study, there is no definite description of them in literature.

Firstly, the maxima of a smoothed density field were considered, instead of considering galaxies. These maxima are less numerous than galaxies, and they can be sorted according to the values of their densities. Some limiting values of density can be selected to mark maxima that best trace the distribution of the filaments. The distribution of maxima is shown in Fig. 1. In this work, the angular resolution of $0.1^{\circ}$ was used in all plots. The task of combination of maxima into the filaments, and distinction of different filaments from one another, remains. Note that the distribution of voids in a layer shows that the characteristic size of a void in the local Universe is less than $100 \mathrm{Mpc}$.

\section{MINIMAL GRADIENT LINES}

Let's consider the following model of LSS presentation in the layer of smoothed density. Suppose that the intersections of filaments correspond to galaxy clusters and are the largest maxima of galaxy density. The most simple, natural and intuitively understandable case is the intersection of three filaments in each cluster. If all filaments in the layer have the same size and all angles between the intersecting filaments are equal to $120^{\circ}$, we will have a hexagonal $2 \mathrm{D}$ grid of filaments and voids. Such a pattern characterizes the plane but not three-dimensional space. In this toy model the lines of maximum density should pass from a cluster along the middle line of a filament to a void. Such lines pass starting from density maxima to the direction of a minimal density gradient. Also we should take less maxima than in Fig. 1. The ideal variant of the application of such a method will take three very nearby maxima at each filament intersection. The number of maxima can be decreased by selecting the largest maximum in a circle of some fixed radius. Gradient lines that were obtained in such a way absolutely do not correspond to the distribution of visible filaments, so we will not show them here at all. The explanation of this inconsistency may be the following. Firstly, maxima appear not only at filament intersections, but also often in any places in a filament. Secondly, even in the most optimal case, when a gradient line passes from a maximum along a filament, it always meets another line passing from the opposite end of a filament. A saddle-like shape of a smoothed density field appears in the middle of a filament and both gradient lines fall onto a void. The process of selection of the minimal gradient direction is very unstable, so almost all gradient lines fall onto voids perpendicular to the filaments. If there was a much larger number of galaxies, the gradient lines would trace the filaments as their normal's in all points. But the real number of galaxies seems not to be enough for further development of this method.

\section{TREE GRAPH ON SQUARE GRID}

The best detectable filaments are the largest ones, with sizes close to $100 \mathrm{Mpc}$. A fine filamentary structure of galaxy distribution cannot be recovered, because if we consider the task of detection of a small filament, we will soon come across filaments from too few a number of galaxies, that are statistically insignificant. X-ray galaxies can be observed at larger average distances than normal [4, 5]. Having in perspective the task of comparison of a large-scale galaxy distribution in the optical and X-ray bands, 
we are interested in filament detection at larger possible distances. Thus we have to add the main features of filaments to the basis of the method for filament detection. The next method develops the idea of searching for filament intersections, which was described previously. The filament network in a $2 \mathrm{D}$ layer can be presented as a set of intersections, some of which are connected by straight lines (suppose the filaments are not curved). Then we have to find positions of these intersections and prepare a list of intersections which should be connected with every intersection. We should finally get a tree-like graph that covers the sky. We will consider the points of a square grid as possible intersections. The size of a grid cell should be smaller than the size of a void to avoid the skipping of real intersections. Thus the point of such a smaller grid can have up to three connections with neighbours. The point with three connections should be the intersection of three filaments. The point with two connections will appear in the middle of the filament. The points with a single connection should be the tails of lost filaments that do not bound to an intersection. Finally, the points inside the void should not have connections with neighbours at all. An example of such a network of filaments at a square grid is shown in Fig. 2. Grid lines correspond to general features of filament distribution, but the point in a filament is connected with a saw-like line instead of the straight one. The plot of the entire smoothed density field leads to an unexpected feature. It appeared that a cutoff radius has much more influence on a picture than a dispersion parameter of Gaussian. Moreover, the filaments are more easily detected at a density map with larger dispersion but the same cutoff radius. They are also easily detected for smoothing with a flat window function instead of Gaussian. Thus, in the next work we will present the distribution of galaxies as a set of clusters of the same radius.

\section{CONCLUSION}

The most promising method for filament detection in a layer with a smoothed galaxy density field is the description of LSS as a grid of clusters with density larger than a limited value. The network of filaments can not be obtained as gradient lines of a smoothed density field.

\section{ACKNOWLEDGEMENT}

The author is greatful to the Sloan Digital Sky Survey team. Funding for the SDSS and SDSSII has been provided by the Alfred P. Sloan Foundation, the Participating Institutions, the National Science Foundation, the U.S. Department of Energy, the National Aeronautics and Space Administration, the Japanese Monbukagakusho, the Max Planck Society, and the Higher Education Funding Council for England. The SDSS Web Site is http://www.sdss.org/. The SDSS is managed by the Astrophysical Research Consortium for the Participating Institutions. The Participating Institutions are the American Museum of Natural History, Astrophysical Institute Potsdam, University of Basel, University of Cambridge, Case Western Reserve University, University of Chicago, Drexel University, Fermilab, the Institute for Advanced Study, the Japan Participation Group, Johns Hopkins University, the Joint Institute for Nuclear Astrophysics, the Kavli Institute for Particle Astrophysics and Cosmology, the Korean Scientist Group, the Chinese Academy of Sciences (LAMOST), Los Alamos National Laboratory, the Max-Planck-Institute for Astronomy (MPIA), the Max-Planck-Institute for Astrophysics (MPA), New Mexico State University, Ohio State University, University of Pittsburgh, University of Portsmouth, Princeton University, the United States Naval Observatory, and the University of Washington.

\section{REFERENCES}

[1] Smith A. G., Hopkins A. M., Hunstead R. W. \& Pimbblet K. A. 2012, MNRAS, 422, 25

[2] Sousbie T. 2011, MNRAS, 414, 350

[3] Tempel E., Stoica R.S., Martínez V.J. et al. 2014, MNRAS, 438, 3465

[4] Tugay A. V. 2012, Odessa Astronomical Publications, 25, 142

[5] Tugay A. V. \& Vasylenko A. A. 2011, Odessa Astronomical Publications, 24, 72 


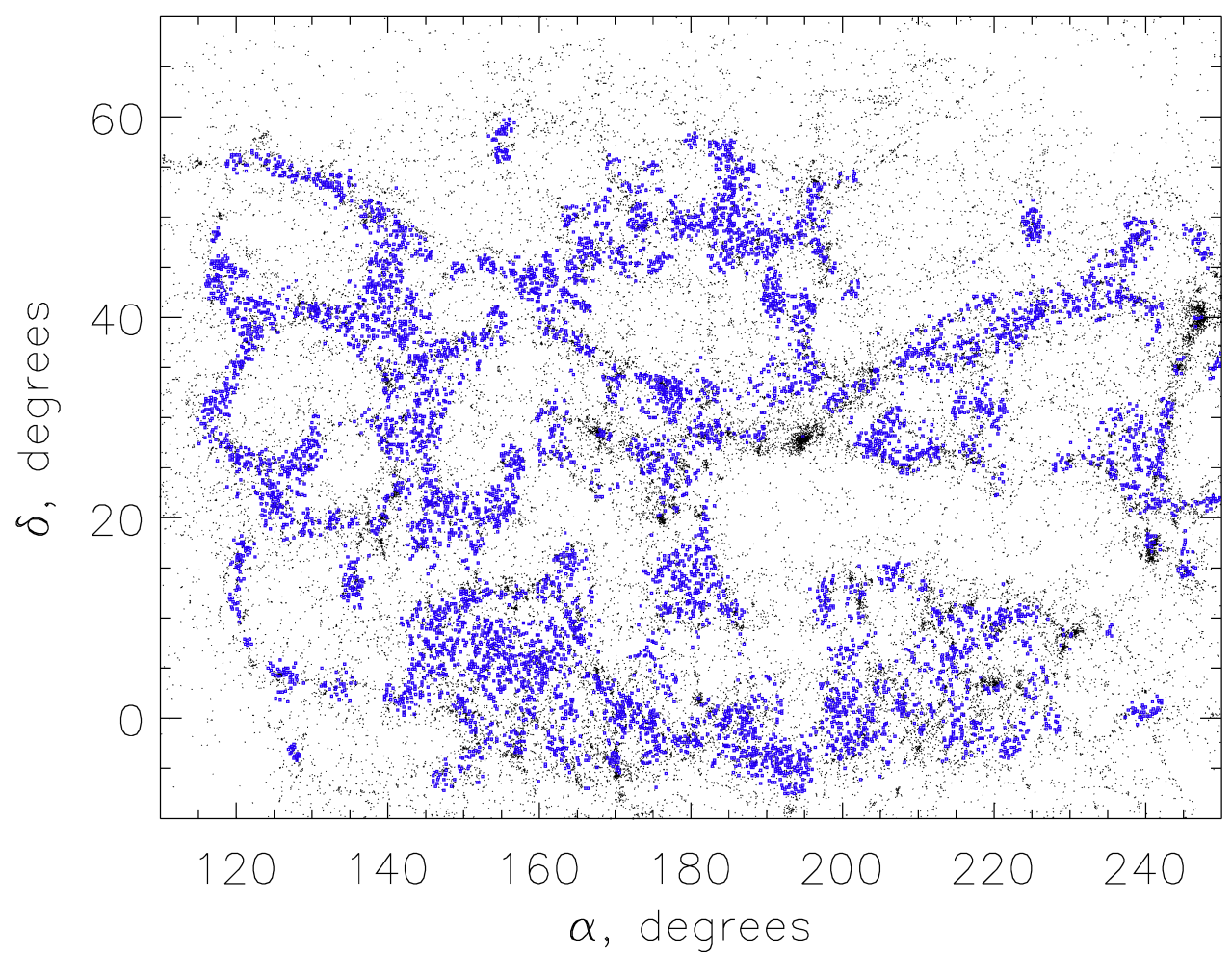

Fig. 1: Sky distribution of SDSS galaxies with radial velocities between 4000 and $11000 \mathrm{~km} / \mathrm{s}$. Large dots are maxima of smoothed density field.

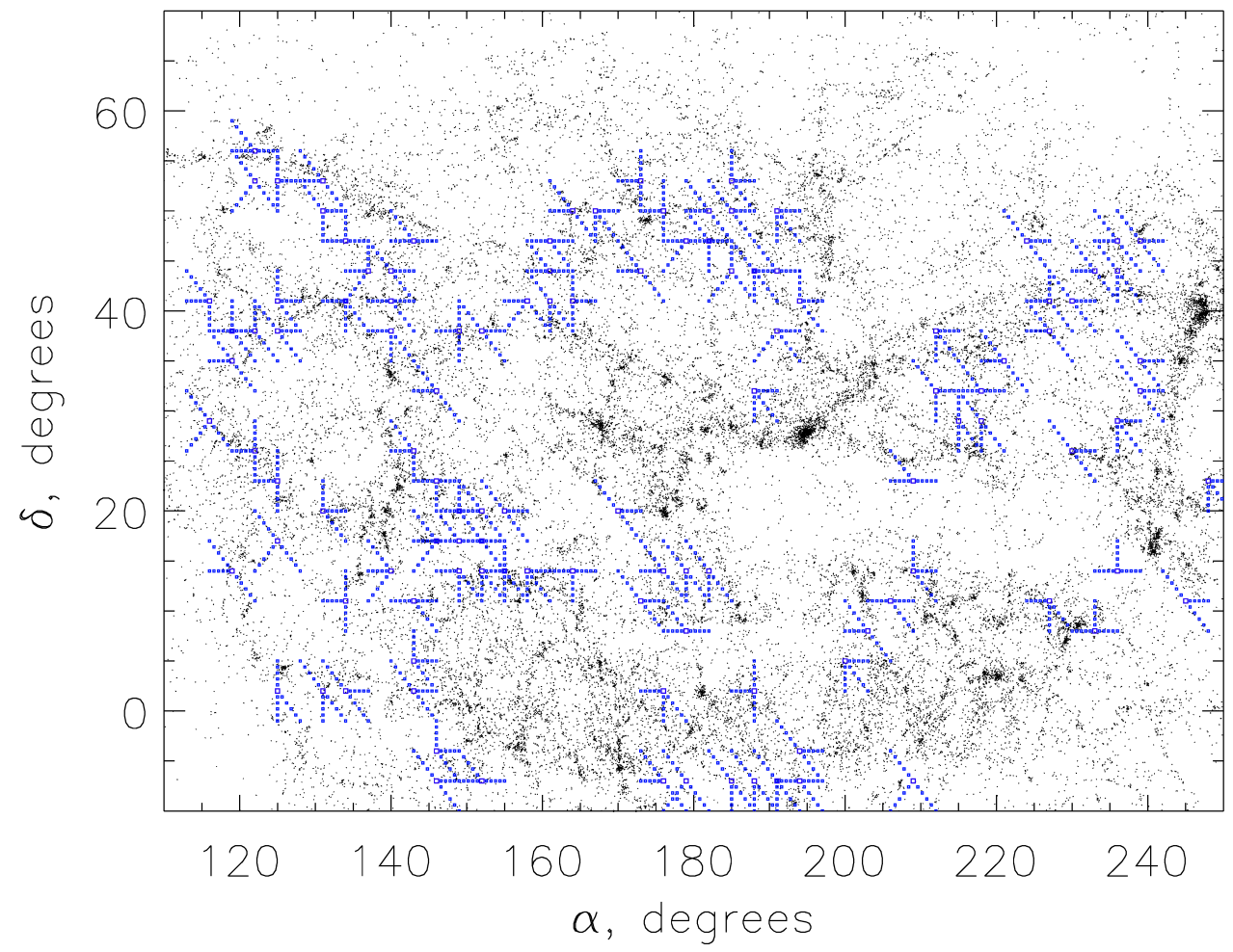

Fig. 2: Filament network on a square grid. Each point of a grid with smoothed density larger than a limiting value is connected with three nearby points with the closest values of galaxy density. 